\title{
Influência do zumbido nos limiares auditivos de altas freqüências
}

\section{Tinnitus influence on high-frequency hearing thresholds}

\author{
Rafaella Bastos Silva Figuerêdo ${ }^{1}$, Ana Paula Corona ${ }^{2}$
}

\begin{abstract}
RESUMO
Objetivo: Estudar a influência do zumbido nos limiares auditivos das altas freqüências. Métodos: A amostra foi composta por 30 indivíduos do sexo feminino, com idades entre 19 a 56 anos, sendo 14 com zumbido (grupo estudo) e 16 sem zumbido (grupo controle), todos com limiares tonais de 0,25 a $8 \mathrm{kHz}$ até $25 \mathrm{~dB}$ NA. Foram pesquisados os limiares tonais de $9,10,12,14$ e 16 kHz. Resultados: Houve diferença estatisticamente significante entre os limiares auditivos de alta frequiência entre o grupo estudo e o controle, sendo piores no grupo estudo. Não foi observada diferença significante entre os limiares auditivos das orelhas direita e esquerda dos indivíduos do grupo estudo, bem como em relação à localização da queixa de zumbido. Observou-se diferença estatisticamente significante entre as médias de idade dos grupos, sendo o grupo estudo composto por pessoas mais velhas. Conclusão: A pesquisa das altas frequiências apresenta-se como uma alternativa diagnóstica na identificação precoce de perdas auditivas.
\end{abstract}

DESCRITORES: Zumbido; Audiometria/métodos; Limiar auditivo/fisiologia

\section{INTRODUÇÃO}

O zumbido é um sintoma que afeta muitas pessoas no mundo inteiro. Na população brasileira, atinge entre $15 \mathrm{e}$ $20 \%$ dos indivíduos ${ }^{(1)}$. Pacientes com queixa de zumbido relatam que esse sintoma causa desconforto e, muitas vezes, interfere no seu desempenho profissional, social e nas relações familiares ${ }^{(2)}$.

O zumbido pode ser definido como percepção consciente de um som que se origina nos ouvidos ou na cabeça do paciente, sem a presença de uma fonte externa geradora desse $\operatorname{som}^{(3)}$. Soma-se a este conceito o fato do zumbido estar associado à diminuição da sensibilidade auditiva ${ }^{(4)}$.

Inúmeras são as hipóteses para explicar a origem do zumbido. Causas otológicas, metabólicas, cardiovasculares, neurológicas, farmacológicas, odontológicas e psicológicas já foram descritas na literatura ${ }^{(5)}$. Para alguns autores, o zumbi-

(1) Especializanda em Audiologia pela União Metropolitana de Educação e Cultura - UNIME - Lauro de Freitas (BA), Brasil.

(2) Pós-graduanda do Programa de Pós-Graduação em Medicina e Saúde da Faculdade de Medicina da Universidade Federal da Bahia - UFBA - Salvador (BA), Brasil; Professora do Curso de Fonoaudiologia da Universidade Federal da Bahia - UFBA - Salvador (BA), Brasil.

Trabalho de conclusão de pós-graduação lato sensu realizado no Curso de Fonoaudiologia da União Metropolitana de Educação e Cultura - UNIME Lauro de Freitas (BA), Brasil para a obtenção do título de Especialista em Audiologia.

Endereço para correspondência: Rafaella Bastos Silva Figuerêdo. Av. Sete de Setembro, 1937/402, Vitória, Salvador - BA, CEP 40080-002. E-mail: rafaellafigueredo@yahoo.com.br

Recebido em: 20/9/2006; Aceito em: 15/2/2007 do surge em decorrência da agressão ao sistema auditivo, em algum momento da vida, a qual acarreta alterações estruturais que perpetuam o sintoma. Já, para outros, o zumbido surge concomitante a processos patológicos, fato que poderia explicar a progressão deste sintoma, sua intermitência ou flutuação e a instalação na orelha contra-lateral ${ }^{(3)}$.

Alguns estudos propõem a possibilidade do zumbido ser provocado por uma pequena anomalia na via auditiva ${ }^{(6)}$, acarretando disfunção temporária ou permanente nessas estrutu$\operatorname{ras}^{(7)}$. Acredita-se, ainda, que o zumbido seria uma consequiência de distúrbios cocleares, que resultariam em um aumento na atividade espontânea das fibras do nervo auditivo, causada por uma hiperatividade das células ciliadas da cóclea $^{(8)}$.

Devido à complexidade da fisiopatologia do zumbido e, por se tratar de um sintoma que pode apresentar grande repercussão na vida do indivíduo, há um crescente interesse em estudá-lo. Atualmente, tem sido observado um enfoque no estudo da sensibilidade auditiva para freqüências acima de $8 \mathrm{kHz}^{(9)}$ e alguns estudos relacionam os resultados obtidos nas altas frequiências com a presença do zumbido ${ }^{(10-11)}$.

A audiometria de alta freqüência é um procedimento audiológico que avalia frequiências a partir de $8 \mathrm{kHz}$, podendo chegar até $20 \mathrm{kHz}$ em alguns audiômetros comercializados ${ }^{(12)}$. A pesquisa das altas frequiências tem assumido um papel de destaque na área de diagnóstico audiológico, uma vez que estas freqüências são as primeiras a serem acometidas, na maioria das doenças que afetam a orelha interna ${ }^{(13)}$.

Estudos têm demonstrado piora dos limiares auditivos de altas freqüências em indivíduos com zumbido, sem que efei- 
tos característicos apareçam na faixa de freqüências convencional $(0,25 \mathrm{a} 8 \mathrm{kHz})^{(9)}$. Estes achados podem explicar a queixa de redução da audição em portadores de zumbido.

A obtenção das respostas auditivas para esta região, por meio da audiometria de altas frequiências, pode contribuir para o conhecimento dos mecanismos fisiológicos que provocam esse sintoma, bem como auxiliar na detecção precoce da perda auditiva. Sendo assim, este trabalho tem como objetivo estudar a influência do zumbido nos limiares auditivos das altas freqüências.

\section{MÉTODOS}

Esta pesquisa, de caráter transversal, foi conduzida com dois grupos, um Grupo Controle (GC) com 14 indivíduos e um Grupo Estudo (GE) com 16 indivíduos, totalizando uma amostra aleatória e estratificada de 30 indivíduos adultos, do sexo feminino, com idades entre 19 e 56 anos.

A seleção dos indivíduos do GE foi realizada por intermédio da análise dos prontuários e audiometrias, dentre a população atendida em uma clínica escola. Já, o GC foi composto por estudantes e funcionários da Instituição. Todos os indivíduos aceitaram participar da pesquisa e assinaram o termo de consentimento, aprovado pelo Comitê de Ética em Pesquisa da Instituição, sob o protocolo no 96/05.

Cabe ressaltar que o tamanho reduzido da amostra foi determinado pelo fato de alguns dos indivíduos, inicialmente elegíveis para o estudo, serem excluídos por falta de dados completos no prontuário, por terem alterado seu contato telefônico ou, ainda, por não terem comparecido para realizar os procedimentos nos dias agendados, mesmo após a terceira convocação. Outro aspecto que deve ser considerado refere-se à amostra ser composta apenas por indivíduos do sexo feminino. Optou-se por excluir o sexo masculino, uma vez que o número de perdas entre os sujeitos selecionados foi elevado e por estudos apontarem que o evento de interesse ocorre independente do sexo.

Todos os indivíduos selecionados para o estudo apresentavam limiares auditivos de 0,25 a $8 \mathrm{kHz}$ até $25 \mathrm{~dB}$ NA e curvas timpanométricas do tipo A, com presença dos reflexos acústicos estapedianos contralaterais. Foram excluídos os indivíduos que, na anamnese, relataram exposição atual ou pregressa a ruído e a produtos químicos, uso atual ou pregresso de medicamentos ototóxicos, bem como ausência de antecedentes familiares para perda auditiva, doenças otológicas, alterações metabólicas e/ou cardiovasculares, já que estes fatores podem causar danos cocleares.

No GE, além dos critérios anteriormente mencionados, foram incluídos indivíduos que possuíam zumbido como primeira queixa auditiva.

Todos os indivíduos que atenderam aos critérios de inclusão foram submetidos à anamnese, meatoscopia e audiometria tonal convencional (sendo pesquisadas as freqüências pertencentes à faixa de 0,25 a $8 \mathrm{kHz}$ ). Em seguida, foi realizada a audiometria de alta frequiência, na qual foram pesquisados os limiares auditivos em dB NA nas freqüências de 9 a $16 \mathrm{kHz}$ (Quadro 1). Para a pesquisa dos limiares auditivos foi utilizado o audiômetro modelo AC40, da marca Interacoustics, calibrado para uso com fones de ouvido do tipo TDH-29 na audiometria convencional e do tipo HV/Pro na audiometria de altas freqüências. $\mathrm{O}$ equipamento tem correções para Nível de Audição de acordo com padrões ANSI $3.6-1989$.

Para obtenção dos limiares auditivos de 0,25 a $16 \mathrm{kHz}$, foi aplicada a técnica mista. Considerou-se limiar auditivo a menor intensidade, na qual o indivíduo apresentou $50 \%$ das respostas. Utilizou-se o estímulo sonoro apresentado sob a forma de tom pulsátil, por ser mais facilmente percebido pelo indivíduo portador de zumbido ${ }^{(15)}$.

$\mathrm{Na}$ audiometria de altas frequiências, primeiramente, os tons foram apresentados aos indivíduos, visando à familiarização ao estímulo. Em seguida, foi realizada a pesquisa dos limiares, alternando a orelha para cada participante, isto é, se no primeiro indivíduo a pesquisa fosse iniciada na orelha direita, o próximo seria na esquerda. A estratégia de alternar a orelha para os indivíduos da amostra foi realizada com o intuito de minimizar o efeito do aprendizado, isto é, respostas melhores na segunda orelha testada, prevenindo, assim, um possível viés no resultado do teste. Além dessas medidas adotadas em estudo anterior ${ }^{(16)}$, retornou-se a $9 \mathrm{kHz}$ para re-teste, já que esta foi a primeira a ser testada, a fim de que os resultados pudessem revelar, com maior precisão, a realidade sobre os limiares auditivos de altas freqüência.

Foi utilizado o teste estatístico de Mann-Whitney para verificar se existia diferença entre os limiares auditivos de altas frequiências dos indivíduos do GE e do GC, no que se refere à idade. $\mathrm{O}$ teste de postos com sinais de Wilcoxon foi utilizado para comparar os limiares auditivos entre as orelhas testadas. Já o teste de Kruskal-Wallis foi empregado para verificar a diferença entre os limiares auditivos de altas freqüências dos sujeitos do GE de acordo com a localização do zumbido (bilateral, unilateral à direita e unilateral à esquerda).

\section{RESULTADOS}

Foram avaliados 30 indivíduos, do sexo feminino, subdivididos nos grupos controle e estudo. Todos apresentaram limiares auditivos, na faixa de 250 a $8000 \mathrm{~Hz}$, até $25 \mathrm{~dB}$ NA. O GC foi composto por 16 indivíduos, com idade entre 22 e 55 anos. Já o GE foi constituído por 14 indivíduos, com idade entre 19 a 56 anos.

As Tabelas 1 e 2 indicam os limiares auditivos destes

Quadro 1. Saída mínima e máxima nas freqüências de 9 a 16kHz do audiômetro AC40 - Interascoustic, calibrado em dBNA ${ }^{(14)}$

\begin{tabular}{|lccccc|}
\hline Saída & $\mathbf{9 0 0 0 H z}$ & $\mathbf{1 0 0 0 0 H z}$ & $\mathbf{1 2 0 0 0 H z}$ & $\mathbf{1 4 0 0 0 H z}$ & $\mathbf{1 6 0 0 0 H z}$ \\
\hline Mínima (dBNA) & -10 & -10 & -10 & -10 & -10 \\
Máxima (dBNA) & 105 & 100 & 90 & 85 & 75 \\
\hline
\end{tabular}


Tabela 1. Análise descritiva e comparativa dos limiares auditivos (em dBNA) da orelha direita nas freqüências de 9 a $16 \mathrm{kHz}$ dos sujeitos dos grupos controle e estudo

\begin{tabular}{|c|c|c|c|c|c|c|c|c|}
\hline Freqüência & Grupos & Média & Mediana & Desvio Padrão & Mínimo & Máximo & Tamanho & $p$-valor \\
\hline \multirow[t]{2}{*}{$9 \mathrm{kHz}$} & Controle & 13,44 & 15,00 & 7,465 & -5 & 20 & 25 & 0,0139 \\
\hline & Estudo & 23,57 & 22,50 & 10,995 & 10 & 50 & 40 & \\
\hline \multirow[t]{2}{*}{$10 \mathrm{kHz}$} & Controle & 16,25 & 15,00 & 9,037 & 5 & 40 & 35 & 0,0318 \\
\hline & Estudo & 26,07 & 22,50 & 15,833 & 5 & 70 & 65 & \\
\hline \multirow[t]{2}{*}{$12 \mathrm{kHz}$} & Controle & 15,00 & 15,00 & 8,367 & 0 & 30 & 30 & 0,0200 \\
\hline & Estudo & 31,43 & 22,50 & 20,609 & 10 & 75 & 65 & \\
\hline \multirow[t]{2}{*}{$14 \mathrm{kHz}$} & Controle & 24,69 & 27,50 & 12,841 & 5 & 40 & 35 & 0,0048 \\
\hline & Estudo & 47,50 & 47,50 & 22,850 & 10 & 75 & 65 & \\
\hline \multirow[t]{2}{*}{$16 \mathrm{kHz}$} & Controle & 34,06 & 37,50 & 16,352 & 10 & 70 & 60 & 0,0200 \\
\hline & Estudo & 52,86 & 62,50 & 21,187 & 20 & 75 & 55 & \\
\hline
\end{tabular}

* Teste estatístico de Mann-Whitney

Tabela 2. Análise descritiva e comparativa dos limiares auditivos (em dBNA) da orelha esquerda nas freqüências de 9 a $16 \mathrm{kHz}$ dos sujeitos dos grupos controle e estudo

\begin{tabular}{|c|c|c|c|c|c|c|c|c|}
\hline Freqüência & Grupos & Média & Mediana & Desvio Padrão & Mínimo & Máximo & Tamanho & $\mathrm{p}$-valor \\
\hline \multirow[t]{2}{*}{$9 \mathrm{kHz}$} & Controle & 8,75 & 10,00 & 7,416 & -5 & 20 & 25 & 0,0009 \\
\hline & Estudo & 21,07 & 20,00 & 10,594 & 5 & 50 & 45 & \\
\hline \multirow[t]{2}{*}{$10 \mathrm{kHz}$} & Controle & 15,63 & 15,00 & 9,106 & 5 & 40 & 35 & 0,0151 \\
\hline & Estudo & 28,93 & 27,50 & 17,778 & 5 & 75 & 70 & \\
\hline \multirow[t]{2}{*}{$12 \mathrm{kHz}$} & Controle & 13,13 & 12,50 & 7,500 & 0 & 30 & 30 & 0,0004 \\
\hline & Estudo & 31,43 & 22,50 & 20,609 & 10 & 75 & 65 & \\
\hline \multirow[t]{2}{*}{$14 \mathrm{kHz}$} & Controle & 19,06 & 17,50 & 11,579 & 0 & 45 & 45 & 0,0053 \\
\hline & Estudo & 45,71 & 45,00 & 24,796 & 5 & 75 & 70 & \\
\hline \multirow[t]{2}{*}{$16 \mathrm{kHz}$} & Controle & 33,13 & 35,00 & 16,621 & 10 & 70 & 60 & 0,0059 \\
\hline & Estudo & 53,21 & 57,50 & 19,276 & 20 & 75 & 55 & \\
\hline
\end{tabular}

* Teste estatístico de Mann-Whitney

indivíduos, nas altas freqüências, para as orelhas direita e esquerda, respectivamente. Observou-se que, para ambas as orelhas, houve diversidade de respostas em cada freqüência, principalmente no GE, entre os valores: mínimo e máximo obtidos. Foi verificado, também que, quanto mais alta a freqüência pesquisada, pior era o limiar auditivo obtido tanto no GE quanto no GC.

O GE apresentou limiares auditivos maiores que os do $\mathrm{GC}$, em todas as frequiências pesquisadas para orelhas direita e esquerda $(\mathrm{p}<0,0500)$. No entanto, para a frequiência de $14.000 \mathrm{~Hz}$, foi observado que as diferenças obtidas entre os grupos foram mais significantes na orelha esquerda, ou seja, nesta orelha foi maior a diferença entre os limiares auditivos dos GC e GE.

Os limiares auditivos das altas frequiências foram agrupados e, desta forma, pode-se estabelecer a diferença entre a média dos limiares dos grupos, para cada orelha testada. Esta análise revelou que o grupo de estudo apresentou limiares auditivos médios maiores que o grupo controle $(\mathrm{p}<0,0500)$. Os resultados indicaram, conforme observado na maioria das freqüências pesquisadas, que a diferença entre os grupos foi mais significante na orelha esquerda do que na direita (Tabela 3 ).

Estudou-se, ainda, a sensibilidade auditiva das orelhas direita e esquerda por meio da análise intra-grupo. Foi observado que no GE não houve diferença estatisticamente significante nos valores médios dos limiares auditivos entre os lados direito e esquerdo de cada indivíduo ( $p=0,7232)$.

A análise da influência da idade nos resultados obtidos foi realizada, comparativamente, entre a média da idade de ambos os grupos. Por meio desta, foi possível verificar que existiu diferença significante entre os dois grupos (Tabela 4), sendo o GE composto por pessoas mais velhas ( $\mathrm{p}=0,0081$ ).

Apesar da amostra reduzida do estudo, observou-se a interferência da idade sobre os limiares auditivos, sendo evidencia-

Tabela 3. Distribuição das médias dos limiares auditivos (em dBNA) das orelhas direita e esquerda nos grupos controle e estudo

\begin{tabular}{lcccccccc}
\hline Freqüência & Grupos & Média & Mediana & Desvio Padrão & Mínimo & Máximo & Tamanho & $\mathrm{p}$-valor \\
\hline Orelha direita & Controle & 20,68 & 22,50 & 8,005 & 7 & 33 & 26 & 0,0088 \\
& Estudo & 36,28 & 36,50 & 15,730 & 13 & 68 & 55 \\
Orelha esquerda & Controle & 17,93 & 21,00 & 8,003 & 5 & 34 & 29 \\
& Estudo & 37,42 & 39,00 & 16,04 & 14 & 70 & 56 \\
\hline
\end{tabular}

* Teste estatístico de Mann-Whitney 
Tabela 4. Distribuição das idades dos sujeitos dos grupos controle e estudo

\begin{tabular}{lcc}
\hline Idades & Controle & Estudo \\
\hline Média & 28,38 & 40,38 \\
Mediana & 23,94 & 42,50 \\
Desvio Padrão & 11,915 & 8,326 \\
Mínimo & 22 & 19 \\
Máximo & 55 & 55 \\
Tamanho & 33 & 36 \\
p-valor & & 0,0081 \\
\hline
\end{tabular}

* Teste estatístico de Mann-Whitney

da uma diminuição da sensibilidade auditiva, com o aumento dos limiares em todas as freqüências, maior no GE. Apesar dos indivíduos serem classificados clinicamente como audiologicamente normais, a análise separou os GC e GE, indicando limiares de altas freqüências estatisticamente distintos.

Além disso, foi realizada a comparação entre os limiares em altas freqüências dos indivíduos do GE, segundo a localização de zumbido, buscando identificar se este fator influenciava nos valores dos limiares auditivos de altas freqüências. Não foi evidenciada diferença entre os limiares auditivos médios, tanto para orelha direita, quanto para a orelha esquerda. Desta forma, os resultados obtidos no GE independem da condição do zumbido (Tabela 5).

\section{DISCUSSÃO}

Os indivíduos com zumbido apresentaram limiares auditivos em altas freqüências piores do que os do grupo sem zumbido. Não foi observada diferença significante entre os limiares auditivos das orelhas direita e esquerda dos indivíduos do grupo estudo, bem como em relação à localização da queixa de zumbido.

Este estudo apresentou as respostas auditivas de altas freqüências em indivíduos do gênero feminino. Apesar de ter sido realizado somente com mulheres, a literatura ${ }^{(9,16-17)}$ refere que não há diferença significante entre sexo. Cabe ressaltar que estudos em pacientes com zumbido têm sido realizados em casuística envolvendo apenas um dos gêneros ${ }^{(18-19)}$.

Não foi evidenciada diferença estatisticamente significante entre os limiares auditivos das orelhas: direita e esquerda. Este achado é consistente com outros estudos que usaram metodologia semelhante ${ }^{(11,13,20)}$.

Foi possível verificar diferenças nos limiares auditivos entre os indivíduos do GE e GC para todas as freqüências pesquisadas $(9,10,12,14 \mathrm{e} 16 \mathrm{kHz})$, sendo os limiares auditivos em nível de intensidade mais elevado no grupo com zumbido. Alguns estudos ${ }^{(10-11)}$ corroboram este achado, evidenciando um maior comprometimento destes limiares em indivíduos com queixa de zumbido.

$\mathrm{O}$ zumbido pode estar relacionado à presença de células ciliadas externas danificadas e células ciliadas internas normais, e as populações de células ciliadas externas mais susceptíveis à lesão são aquelas localizadas na região mais basal da cóclea ${ }^{(11)}$. A ocorrência de zumbido em indivíduos sem perda auditiva, também, poderia ser explicada pelo dano difuso de até $30 \%$ das células ciliadas externas em toda a espiral da cóclea, sem haver o comprometimento do limiar auditivo $^{(21)}$. Esses fatores descritos na literatura possibilitam inferir que a presença do zumbido na população estudada poderia estar influenciando na elevação dos limiares auditivos das altas freqüências.

Outro dado observado se refere à tendência de piora dos limiares com o aumento da frequiência, em ambos os grupos. Esse achado, curva audiométrica com configuração descendente, é compatível com o da literatura pesquisada ${ }^{(8,11,17,22)} \mathrm{e}$ pode ser explicado pelo fato da orelha humana necessitar de maior energia sonora para detectar sons de frequiências mais $\operatorname{altas}^{(16)}$. No entanto, o acentuado comprometimento nas freqüências de 14 e 16 kHz sugere um efeito adicional na função coclear, elevando significativamente os limiares auditivos nestas freqüências no grupo com zumbido. Cabe salientar que o efeito idade pode ter contribuído para este comportamento, já que foi evidenciado estatisticamente que o GE era composto por pessoas mais velhas (Tabela 4).

Alguns estudos ${ }^{(8,21)}$ realizados destacam que o comprometimento em altas frequiências aumenta a cada década, a partir dos 20 anos de idade, e que essa mudança é consideravelmente maior nas freqüências de 14, 16 e $18 \mathrm{kHz}$. Este achado é condizente com os dados desta pesquisa, em que se observou, pela análise do desvio padrão e dos limiares auditivos mínimos e máximos obtidos no GE, uma grande variabilidade, principalmente para as frequiências mais altas, 14 e $16 \mathrm{kHz}$. Desta forma, a diferença entre os grupos nos limiares de altas frequiências pode ser atribuída à queixa de zumbido e à idade do GE.

\section{CONCLUSÃO}

Com base na análise dos resultados obtidos no presente

Tabela 5. Distribuição das médias dos limiares auditivos (em dBNA) das orelhas direita e esquerda no grupo estudo segundo a localização de zumbido bilateral, unilateral à direita e unilateral à esquerda

\begin{tabular}{lcccccccc}
\hline Média & Localização & Média & Mediana & Desvio Padrão & Mínimo & Máximo & Tamanho & $p$-valor \\
\hline \multirow{3}{*}{ OD } & Bilateral & 33,14 & 32,00 & 15,08 & 16,00 & 54,00 & 38,00 \\
& Unilateral à D & 40,00 & 38,00 & 6,24 & 35,00 & 47,00 & 12,00 \\
& Unilateral à E & 39,00 & 37,50 & 23,33 & 13,00 & 68,00 & 55,00 \\
\multirow{4}{*}{ OE } & Bilateral & 32,57 & 35,00 & 17,36 & 14,00 & 54,00 & 40,00 \\
& Unilateral à D & 35,33 & 38,00 & 6,42 & 28,00 & 40,00 & 12,00 \\
& Unilateral à E & 47,50 & 45,50 & 17,01 & 29,00 & 70,00 & 41,00 \\
\hline
\end{tabular}

* Teste estatístico de Kruskal-Wallis 
estudo, com relação ao comportamento auditivo nas frequiências de 9 a $16 \mathrm{kHz}$ em indivíduos com queixa de zumbido, conclui-se que:

a) houve diferença significante entre os limiares auditivos de altas freqüências dos indivíduos do grupo estudo e dos indivíduos do grupo controle;

b) não houve diferença significante em relação à variável orelha testada;

c) não foi evidenciada diferença significante entre os limiares de altas freqüências dos indivíduos do grupo estudo, segundo a localização do zumbido, bilateral, unilateral à direita e unilateral à esquerda;

d) a interferência da idade nos limiares auditivos das altas freqüências no grupo estudo não permite afirmar que a queixa do zumbido influenciou na elevação destes limiares. Porém, estas variáveis analisadas em conjunto sugerem uma redução no nível de atividade de células ciliadas da região basal da cóclea, revelando a pesquisa das altas freqüências como uma alternativa diagnóstica na identificação precoce de perdas auditivas.

\begin{abstract}
Purpose: To study tinnitus influence on high-frequency hearing thresholds (HFHTs). Methods: The sample consisted of 30 female subjects aged 19 to 56 years, 14 with reported tinnitus (study group) and 16 with no tinnitus complaints (control group). All subjects had pure tone thresholds up to $25 \mathrm{~dB}$ in the frequency range from 0.25 through $8 \mathrm{kHz}$. The research investigated the pure tone thresholds of 9,10,12,14 and $16 \mathrm{kHz}$. Results: The study group was found to have significantly lower HFHTs. No significant differences were found between hearing thresholds for right and left ears in the study group, as well as regarding the reported localization of the tinnitus. The average age of the subjects in the study group was significantly higher when compared to the control group. Conclusion: Determination of HFHTs is a diagnostic alternative for an early identification of hearing losses.
\end{abstract}

KEYWORDS: Tinnitus; Audiometry/methods; Auditory threshold/physiology

\title{
REFERÊNCIAS
}

1. Jastreboff PJ, Jastreboff MM. Tinnitus Retraining Therapy (TRT) as a method of tinnitus and hyperacusis patients. J Am Acad Audiol. 2000;11(3):162-77.

2. Caovilla HH, Silva MLG, Munhoz MSL, Ganança MM. Entendendo as tonturas: o que você precisa saber sobre os distúrbios do labirinto. São Paulo: Atheneu; 1999. 90p.

3. Samelli AG. Zumbido: avaliação, diagnóstico e reabilitação (abordagens atuais). São Paulo: Lovise; 2004. 136p.

4. Sanchez TG, Balbani APS, Bittar RSM, Bento RF. Teste da lidocaína em pacientes com zumbido: quando realizar e como interpretar. Arq Fund Otorrinolaringol. 1998;2(1):1-6.

5. Sanchez TG, Zonato AY, Bittar RSM, Bento RF. Controvérsias sobre a fisiologia do zumbido. Arq Fund Otorrinolaringol. 1997;1(1):2-8.

6. Olsen S0. Zumbido: Resultado da exposição a níveis sonoros excessivos. ln: Nudelmann AA, Costa EA, Seligman J, Ibañez N, organizadores. PAIR: perda auditiva induzida pelo ruído. Rio de Janeiro: Revinter; 2001. vol. 2. p. 93-105.

7. Möller AR. Pathophysiology of tinnitus. Ann Otol Rhinol Laryngol. 1984;93(1 Pt 1):39-44. Review.

8. Pedalini MEB, Sanchez TG, D'Antonio A, D'Antonio W, Balbani A, Hachiya $\mathrm{A}$, et al. Média dos limiares tonais na audiometria de alta frequiência em indivíduos normais de 4 a 60 anos. Pró-Fono. 2000;12(2):17-20.

9. Azevedo LL, Iorio MCM. Estudo dos limiares de audibilidade nas altas freqüências em indivíduos de 12 a 15 anos com audição normal. Acta AWHO. 1999;18(2):78-85.

10. Cai Y, Tang J, Li X. [Relationship between high frequency hearing threshold and tinnitus]. Lin Chuang Er Bi Yan Hou Ke Za Zhi. 2004;18(1):8-9,11. Chinese.

11. Burguetti FAR, Peloggia AG, Carvallo RMM. Limiares de audibilidade em altas frequiências em indivíduos com queixa de zumbido. Arq Intern Otorrinolaringol [periódico na Internet]. 2004 [citado $2006 \mathrm{Jul}$ 19];8(4):[cerca de 7p.]. Disponivel em: http://www.arquivosdeorl.org.br/ conteudo/acervo_port.asp?id=292
12. Frazza MM, et al. Audiometria tonal e vocal. In: Munhoz MSL, Caovilla HH, Silva MLG, editores. Audiologia clínica. São Paulo: Atheneu; 2003. 284p.

13. Carvallo RMM, Koga MC, Carvalho M, Ishida IM. Limiares auditivos para altas freqüências em adultos sem queixa auditiva. Acta AWHO. 2002; 21(1).

14. AC40 Instruction for use - English. Manual ac40 [text on the Internet]. 1997. Arkansas. Audiometric Corporation; c2002. Available form: http:/ /www.audiometrics.com/ac40spec.htm.

15. Figuerêdo RBS. Estudo dos limiares para altas freqüências em indivíduos com audição normal [monografia]. Salvador: Universidade Federal da Bahia; 2004.

16. Sahyeb DR, Costa Filho OA, Alvarenga KF. Audiometria de alta freqüência: estudo com indivíduos audiologicamente normais. Rev Bras Otorrinolaringol. 2003;69(1):93-9.

17. Zeigelboim BS, Fukuda Y, Iorio MCM. Audiometria de alta freqüência. Acta AWHO. 1996;15(3):155-8.

18. Fernandes JB, Mota HB. Estudo dos limiares de audibilidade nas altas freqüências em trabalhadores expostos a ruído e solvente. Pró-Fono. 2001;13(1):1-8.

19. Samelli AG, Schochat E. Estudo das emissões otoacústicas em pacientes com zumbido. Pró-Fono. 2002;14(1):99-110.

20. Laitila P, Karma P, Sipila M, Manninen M, Rakho T. Extended high frequency hearing and history of acute otitis media in 14-year-old children in Finland. Acta Otolaryngol Suppl. 1997;529:27-9.

21. Ahmed HO, Dennis JH, Badran O, Ismail M, Ballal SG, Ashoor A, Jerwood D. High-frequency (10-18 kHz) hearing thresholds: reliability, and effects of age and occupational noise exposure. Occup Med (Lond). 2001;51(4):245-58.

22. Beltrami CHB. Dos limiares de audibilidade nas frequiências de 250 a $18000 \mathrm{~Hz}$ em indivíduos expostos a ruído ocupacional [tese]. São Paulo: Universidade Federal de São Paulo; 1999. 